\title{
The combined use of order tracking techniques for enhanced Fourier analysis of order components
}

\author{
K. S. Wang and P. S. Heyns \\ Dynamic Systems Group, Department of Mechanical and Aeronautical Engineering, \\ University of Pretoria, Pretoria, 0002, South Africa \\ Corresponding author: KeSheng Wang (Tel:(+27)12 4203724; Fax:(+27)12 \\ 3625087 Email:keshengwang@gmail.com)
}

\begin{abstract}
Order tracking is one of the most important vibration analysis techniques for diagnosing faults in rotating machinery. It can be performed in many different ways, each of these with distinct advantages and disadvantages. However, in the end the analyst will often use Fourier analysis to transform the data from a time series to frequency or order spectra. It is therefore surprising that the study of the Fourier analysis of order-tracked systems seems to have been largely ignored in the literature. This paper considers the frequently used Vold-Kalman filter-based order tracking and computed order tracking techniques. The main pros and cons of each technique for Fourier analysis are discussed and the sequential use of Vold-Kalman filtering and computed order tracking is proposed as a novel idea to enhance the results of Fourier analysis for determining the order components. The advantages of the combined use of these order tracking techniques are demonstrated numerically on an SDOF rotor simulation model. Finally, the approach is also demonstrated on experimental data from a real rotating machine.
\end{abstract}

Keywords: Computed order tracking (COT), Fourier analysis, Order tracking (OT), Rotating machinery, Vold-Kalman filtering and computed order tracking (VKC-OT), Vold-Kalman filter-based order tracking (VKF-OT).

\section{Introduction}

Order tracking (OT) is one of the most important vibration analysis techniques for diagnosing faults in rotating machinery. The main advantage of OT over other vibration analysis techniques lies in the analysis of non-stationary noise and vibration, which vary in frequency with the rotation of a reference shaft or shafts. Order domain analysis relates the vibration signal to the rotating speed of the shaft, instead of to an absolute frequency base. In this way, the vibration components that are proportional to multiples of the running speed can easily be identified. OT can be performed in many different ways, each of these with distinct advantages and disadvantages. 
Among the OT techniques, two approaches are frequently employed. The first is angle domain sampling-based order tracking (AD-OT) or computed order tracking (COT). Various papers discussing the theory and implementation of this approach are available (see for example the work by Fyfe and Munk [1]). The significance of AD-OT or COT is that the re-sampled data have the same properties as stationary frequency data, but the data still need to be processed further by Fourier analysis in terms of uniform angular intervals, instead of uniform time intervals. The re-sampling process is performed, however, subject to artificial assumptions for re-sampling the data, therefore unavoidable errors are introduced. Although COT has some limitations due to the re-sampling and the Fourier analysis process, it remains a very useful tool for analyzing rotating machinery signals in industry, as has indeed been demonstrated by authors such as Eggers et al. [2].

Another approach is what is called waveform reconstruction OT, a typical example of which is Vold-Kalman filter-based order tracking (VKF-OT). This approach to OT can overcome many of the limitations of other OT techniques, such as allowing the high-performance tracking of harmonic responses or orders, and allowing the beat-free extraction of close and crossing orders. Compared to COT, therefore, it captures much shorter transients with no phase bias or slew rate limitations [3]. One of the major differences of this approach compared to the others is that the time signal corresponding to a specific order can be extracted from the raw data, with its amplitude and phase. This comparatively new order tracking technique has been studied over the past ten years [4, 5, and 6]. The characteristics of Vold-Kalman filters were also considered by Herlufsen et al. [7]. Recently, Tůma [5] dealt with the often-neglected issue of setting the filter pass band, which is fundamental to the theory of the MATLAB-based Vibratools scripts [8]. This made the application of VKF-OT more readily accessible to engineers.

Subsequent to the OT itself, Fourier analysis is generally used to transform re-sampled data (i.e. COT) or time series (i.e. VKF-OT) into the order or frequency domains. But the study of Fourier analysis in the context of OT seems to have been ignored in the literature. In fact, meaningful Fourier analysis is relies heavily on the nature of the data to be analyzed. Howell [9] deals comprehensively with the mathematics of Fourier analysis. Most of these theories are derived from the assumptions of continuity, smoothness and periodicity or stationarity. It follows that Fourier analysis is only valid under strict assumptions which, when violated, lead to results that make little physical sense. However, real machine vibration data may not be ideally suited to Fourier analysis. Since real machinery may not behave linearly or may be operating in a noisy environment, the data obtained from these systems may be distorted harmonic waves, or be contaminated with excessive noise, etc. Besides, the machinery may be operating in a non-stationary way (i.e. fluctuating rotating speeds in rotating machinery) and then the measured vibration data will not be periodic or stationary. As a result, the final Fourier analysis figures may appear to be messy. A technique to obtain clear order spectra via Fourier analysis for 
non-stationary and noisy real machinery systems is consequently of great practical importance for the diagnostic process.

A novel approach which combines the use of two different order tracking techniques is presented to deal with this problem. Combining VKF-OT and COT actually exploits the advantages of both order tracking techniques while overcoming their limitations. A simple single-degree-of-freedom rotor system model is then employed to demonstrate the advantages of the proposed technique in simulated environments. Finally, an experimental study is presented, demonstrating the advantages of the proposed technique on an actual rotating machine.

\section{Exploring the two-order-tracking approaches}

Combining the use of the two OT approaches to improve the subsequent Fourier analysis requires an understanding of the nature of these techniques and how their characteristics affect the Fourier analysis.

\subsection{Vold-Kalman filter-based order tracking}

Herlufsen et al. [7] describe order tracking as the art and science of extracting the sinusoidal content of measurements, with the sinusoidal content or orders/harmonics at frequencies that are multiples of the fundamental rotational frequency. To this end, VKF-OT relies on two equations to complete the filtering, namely the data equation and the structural equation. These equations define local constraints, which ensure that the unknown phase assigned orders are smooth and that the sum of the orders should approximate the total measured signal. Tưma [5] mathematically described data and structural equations to investigate the often neglected issue of setting the pass bandwidth in Vold-Kalman filter order tracking. In-depth consideration of the two equations reveals fundamental characteristics of filtered orders from a Vold-Kalman filter. Firstly, the order signal contained in the data equation is the convolution of a complex envelope and a sinusoidally natured complex carrier wave in which rotational frequency is used to determine the complex carrier wave. Due to the possible non-stationary character of rotational speed in rotating machines, the resultant complex carrier wave could be non-stationary and therefore the filtered order will retain its non-stationary nature. Secondly, the structural equation enforces successive digital points of filtered order signal by fitting a low order polynomial. A low order polynomial enforcement to signal sequence is a sufficient condition for smoothness of filtered order [7]. By considering the data and structural equations, one may conclude that the order components extracted from the Vold-Kalman filter are smooth and harmonic waves, but they may be non-stationary.

\subsection{Computed order tracking}

Computed order tracking is a very commonly performed and effective order tracking 
technique. Although inevitable errors will be introduced during the re-sampling process and its artificial assumptions [1], the technique still renders very useful results, and effectively transforms non-stationary time domain data to stationary angular domain data for rotating machinery. Blough [10] uses a graphic representation to explain this transformation process on a simple sine wave. It is clearly demonstrated that the re-sampled data has the same properties as a stationary frequency sine wave sampled at uniform time intervals. This uniformly spaced re-sampled data or stationary re-sampled data can be effectively processed by using the Fourier transform to obtain clear estimates of the orders of interest. This implies a clearer analysis of the signal using the Fourier transform and also indicates that the re-sampling process can be applied to harmonic waves and is not confined to data on rotating machinery vibration. However, COT does not address the quality of the raw data. Imperfections, such as distorted harmonic waves and noise, continue to exist. Besides, COT can only deal with the raw data as a whole and therefore loses the ability to separate each different order from the raw signal.

In paragraph 2.1 it is argued that the Vold-Kalman filter enforces the smoothness as well as the harmonic nature of the filtered data. The harmonic nature does not, however, ensure a stationary harmonic wave, although the re-sampling process can transform data from a non-stationary harmonic wave to a stationary harmonic wave in frequency. This suggests the possibility of using a Vold-Kalman filter to obtain smooth but possibly varying frequency harmonic waves and then transforming them to become stationary in frequency by using the re-sampling process of computed order tracking.

\subsection{Combined use of Vold-Kalman filtering and computed order tracking (VKC-OT)}

The main ideas from paragraphs 2.1 and 2.2 may be summarized as follows:

- Order components from the Vold-Kalman filter are clearly harmonic in nature and smooth waves.

- Order components may be harmonic waves of varying frequency due to the possible of varying rotational speed.

- It can be seen from paragraph 2.2 that the re-sampling process can transform varying frequency harmonic waves to stationary frequency harmonic waves. A Fourier analysis is then used to transform the re-sampled time domain data to the order domain.

Therefore, if data are obtained from a non-stationary and noisy real machinery system and the data are then processed through a Vold-Kalman filter to extract order/orders of interest, and the filtered order signals are subsequently used as an input to COT, one obtains order waves that are smooth, stationary frequency harmonic waves. Under these conditions the stringent requirements of Fourier 
analysis are largely satisfied. One may therefore expect clean and clear order spectra by means of this process. Based upon the above reasoning it follows that if the two order tracking methods are applied in sequence (VKF-OT and then COT), the restrictions of Fourier analysis can be largely satisfied to render clean order spectra. This combined use of order tracking techniques may be referred to as Vold-Kalman filter and computed order tracking (VKC-OT). Figure 1 describes graphically the advantages of the combined use of the two order tracking techniques in sequence.
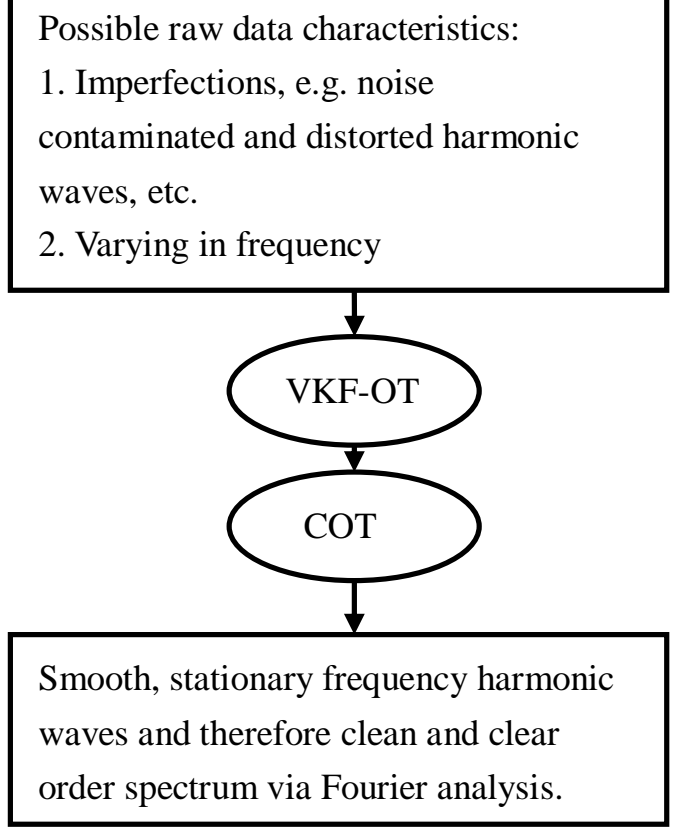

Figure 1 Advantages of VKC-OT

\section{Simulation study}

\subsection{Simulation of a single degree-of-freedom (SDOF) rotor model response}

The following simulation studies illustrate the use of the VKC-OT approach. Since order tracking is primarily used for vibration signals from rotating machines, the first example considers a very simple SDOF rotor system. Here the lateral response of a symmetric rotor is modelled as two uncoupled SDOF systems (see Figure 2). 


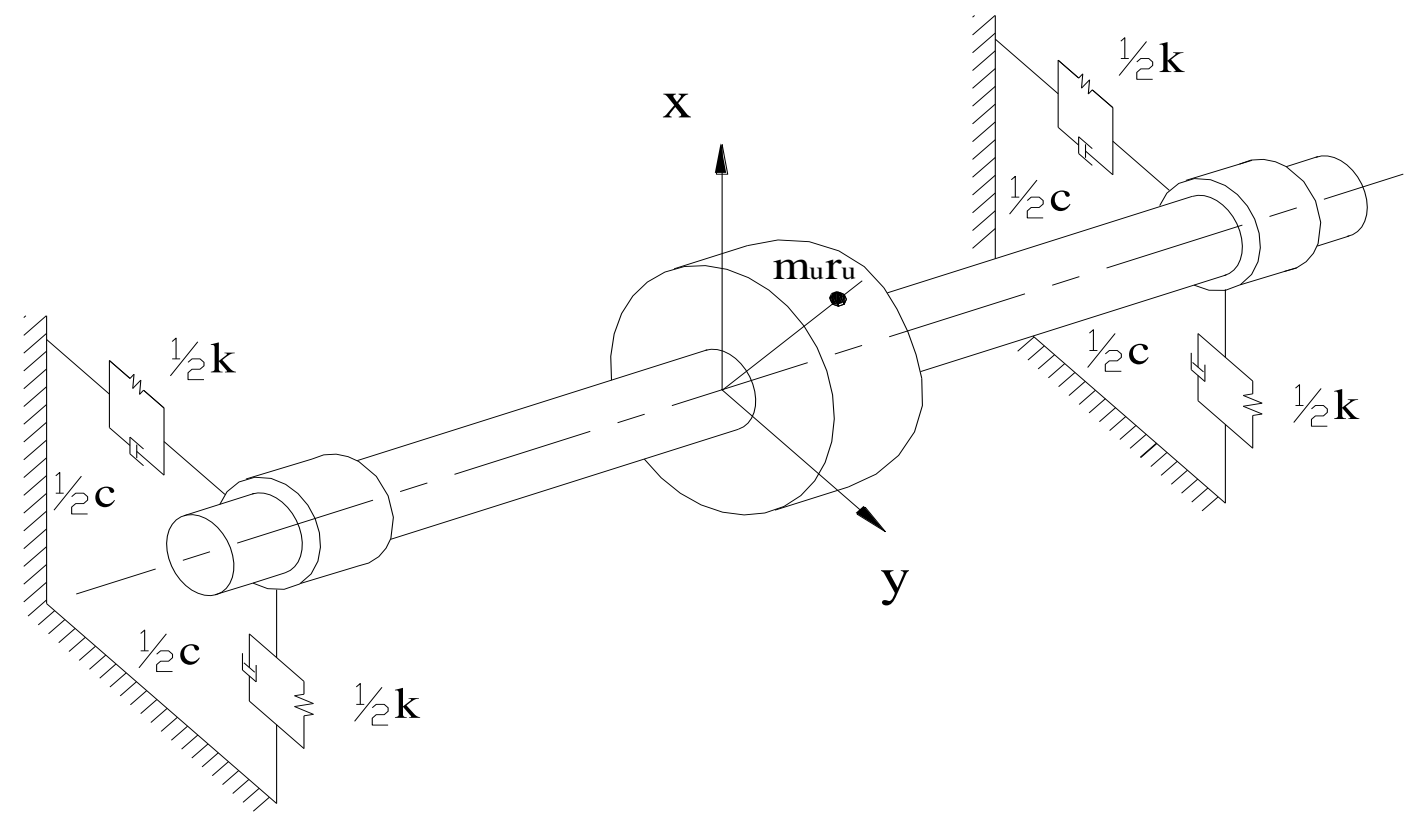

Figure 2 Rotor model

It is assumed that a rotor of mass $\mathrm{m}$ is mounted on bearings of total stiffness $\mathrm{k}$ and damping coefficient $\mathrm{c}$, in both the $\mathrm{x}$ and $\mathrm{y}$ directions. The rotor rotates at an increasing speed. The external excitation forces comprise two harmonic components with quadratically increasing frequency (in each direction). The system response with and without random noise scenarios are studied. Detailed characteristics are shown in Table 1.

Table 1 Characteristics of the SDOF rotor model

\begin{tabular}{|l|l|}
\hline Parameter & Value \\
\hline Rotor mass $m$ & $20 \mathrm{~kg}$ \\
\hline Damping coefficient $c$ & $100 \mathrm{Ns} / \mathrm{m}$ \\
\hline Stiffness $k$ & $500000 \mathrm{~N} / \mathrm{m}$ \\
\hline Eccentricity $r_{u}$ & $0.1 \mathrm{~m}$ \\
\hline Unbalance mass $\mathrm{m}_{\mathrm{u}}$ & $0.05 \mathrm{~kg}$ \\
\hline
\end{tabular}




\begin{tabular}{|l|l|}
\hline Initial time $t_{0}$ & $0 \mathrm{~s}$ \\
\hline Final time $t_{f}$ & $5 \mathrm{~s}$ \\
\hline Time steps & 4096 \\
\hline Number of revolutions & 100 \\
\hline Numples of resampling & 100 \\
\hline Angular speed $\omega$ & $15.0796 t^{2}$ \\
\hline Scenario 1 & Excitation: $F=m_{u} \omega^{2} r_{u} \sin (3 \omega t)+m_{u} \omega^{2} r_{u} \sin (6 \omega t)$. \\
\hline Scenario 2 & $\begin{array}{l}\text { Excitation: } F=m_{u} \omega^{2} r_{u} \sin (3 \omega t)+m_{u} \omega^{2} r_{u} \sin (6 \omega t) \\
\text { Final System response }=\operatorname{system~response~to~} F+\text { Noise } \\
\text { Noise: } 100 \times \text { randn }(4096,1) \\
(\text { normally distributed random noise with mean zero } \\
\text { and standard deviation one). }\end{array}$ \\
\hline
\end{tabular}

Firstly, scenario 1 (orders 3 and 6 only - see Table 1) is considered and the Power Spectral Density (PSD) results of different techniques are presented in Figure 3. 


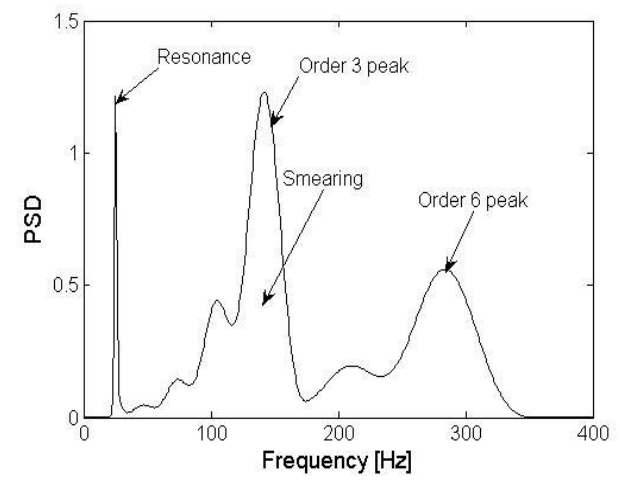

a. PSD on raw data

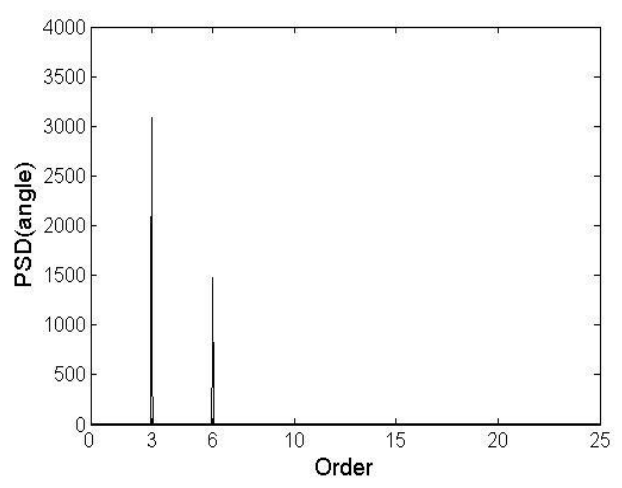

c. PSD on COT data

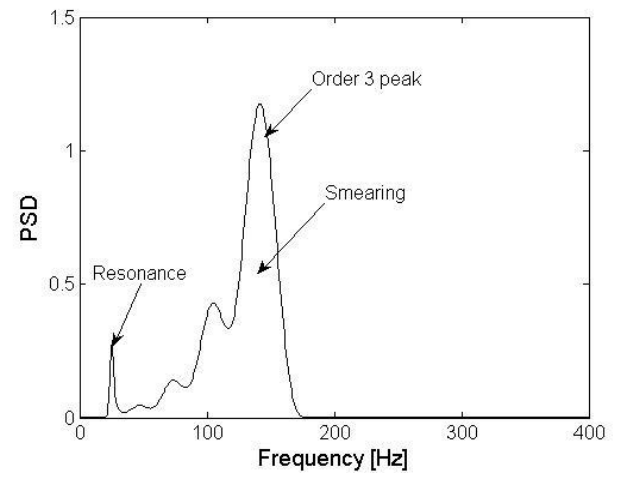

b. PSD on VKF-OT for $3^{\text {rd }}$ order

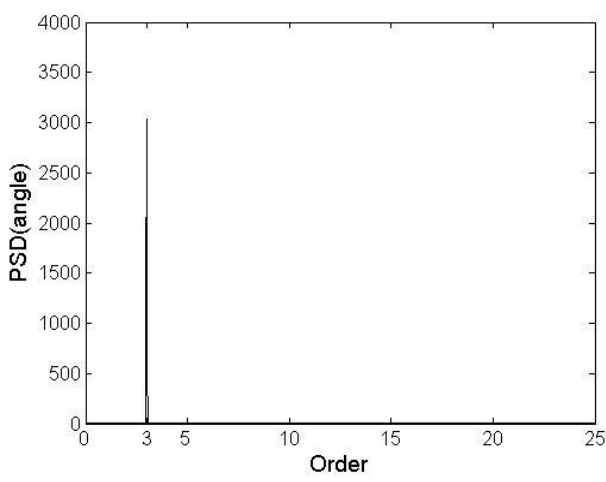

d. PSD on VKC-OT for $3^{\text {rd }}$ order

Figure 3 PSD results for scenario 1 (orders 3 and 6)

In Figure 3(a), which shows the PSD of the system response, there is one sharp resonance peak (fixed time-based frequency) and two rounded order peaks (smearing due to the non-stationarity of the time-based data caused by the increasing excitation frequencies). Figure 3(b) shows the result of performing a PSD on VKF-OT which highlights the $3^{\text {rd }}$ order information and removes the $6^{\text {th }}$ order. The non-stationary smearing effect is still visible in the spectrum. The system resonance is also largely removed. Remnants of the resonance remain because of the $50 \%$ relative filter bandwidth which was used.

The $50 \%$ relative filter bandwidth means that the ratio of the instantaneous absolute filter bandwidth to the instantaneous rotational speed frequency is 0.5 . Here it is necessary to elaborate on the choice of pass band filter for Vold-Kalman filter tracking. One must be very cautious that for the narrower filter bandwidth, the better tracking abilities do not hold. The Vold-Kalman filter obeys a time frequency relationship, $B_{3 d B} \times \tau=0.2$, where $B_{3 d B}$ is the $3 d B$ bandwidth of the Vold-Kalman filter and $\tau$ is the time it takes for the time response to decay by $8.96 d B$ [7]. It should be noticed that the relationship between $B_{3 d B}$ and $\tau$ is inversely proportional. 
Therefore, a narrower filter pass band will result in a longer time response to track the changes of signals. As a result, the narrow pass band reduces the permissible rate of change in rotational speed. The choice of filter bandwidth needs to be done very carefully. For example analysis the reader may refer to [11] and for details on the choice of the filter bandwidth, the reader may refer to [7].

Figure 3(c) displays the result of COT, namely that there are two clear peaks in the order spectrum at the $3^{\text {rd }}$ and $6^{\text {th }}$ orders respectively. This is because the data are being generated for an ideal linear system with sinusoidal external excitation, and because the re-sampling process of COT rearranges the data to become stationary. The data therefore represent stationary harmonic waves which are ideal for Fourier analysis.

It should be noted that the numerical values of the PSDs in Figure 3(a and b) and 3(c and d) differ significantly. Again, here it should be emphasized that the input signal to COT analysis is the filtered order signal from VKF-OT. Since the simulated responses were calculated at 4096 points in time using the model, the filtered $3^{\text {rd }}$ order also contains 4096 time points. The given rotor rotates 100 revolutions within $5 \mathrm{~s}$ and each revolution is evenly sampled with 100 angular intervals, therefore the resultant samples for COT analysis becomes 10000 instead of 4096. Besides, in Figure 3( $a$ and $b$ ) the PSD describes how the energy associated with the time domain series is distributed with frequency. The unit is $\left(\mathrm{m} / \mathrm{s}^{2}\right)^{2} / \mathrm{Hz}$. Figure 3(c and d) however describes how the energy of an angular domain series is distributed with order. Fundamentally the energy associated with a particular order is distributed over a wide range of frequencies on the time domain-based PSD, owing to the non-stationary nature of the rotating speed. However, on an angle domain based PSD the energy associated with a specific order is reflected at only one order value. The unit is $\left(\mathrm{m} / \mathrm{s}^{2}\right)^{2} /$ order. It is actually a sum of the distributed energies in a frequency domain for a specific order. So as not to confuse readers, the units of PSD are not marked in all figures. However, the acronym PSD is marked on the figures of time domain-based Power Spectra Density, whereas the figures of angle domain based PSD are marked with PSD (angle).

It can be seen that Figure 3(c) present clear peaks, but it should still be borne in mind that the re-sampling process of the angle domain-based method changes the number of samples of raw data (in this case 100 samples per revolution were used to resample the data). In this case, the re-sampling is performed under the assumption of zero rotational acceleration within each revolution, in this way re-sampling the signals in each revolution at equal intervals. Note that the value of the samples per revolution is a parameter that the analyzer may choose arbitrarily. As a result, it may render a different number of re-sampled samples as well as a variation in the value of the re-sampled amplitude and may as such also influence the final result in numerical 
values. Therefore, the process should be kept consistent in the re-sampling samples in all applications of the technique once it has been chosen. What is most important, however, is the angle domain based PSD(angle) cleans up the smearing spectrum from frequency domain into order domain and it features distinct and clear amplitude orders.

Besides, it should also be observed that the system resonance can hardly be recognized in Figure 3(c). This is because the re-sampling occurs in the angular domain, which means the sampling interval changes to a constant angle rather than a constant time interval, and the Fourier analysis actually captures only periodic signals with respect to angle instead of time (as is the case for resonance). Thus COT de-emphasizes the system resonance after the re-sampling.

If the VKC-OT procedure suggested in paragraph 2.3 is applied, however, Figure 3(d) is obtained. This figure shows a single clear $3^{\text {rd }}$ order peak in the spectrum, and illustrates the advantage of the combined use of VKF-OT and COT to obtain diagnostic information about a system. Although Figure 3(c) and (d) both clearly indicate the system information, Figure 3(d) separates the individual $3^{\text {rd }}$ order from the raw data, which makes it possible to investigate further the $3^{\text {rd }}$ order information itself and therefore has an advantage compared to Figure 3(c).

In the above simulation, the response data are generated from an ideal linear system with ideal sinusoidal external excitation, which simplifies the interpretation of the spectra. However, if external noise is added to the system response, the resultant observed response data $=$ response to the external force + noise. To illustrate this, the simulation model uses scenario 2 (in Table 1) and the corresponding PSD results for different techniques are now shown in Figure 4. 


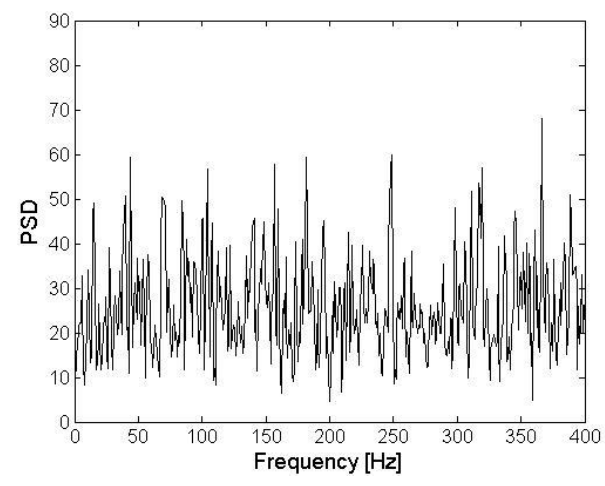

a. PSD on raw data

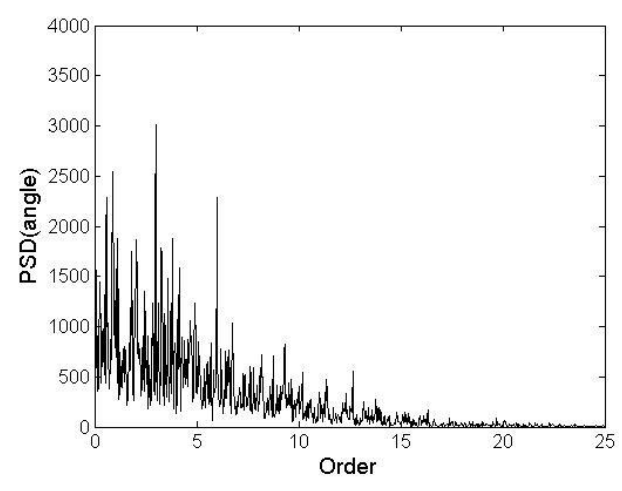

c. PSD on COT data

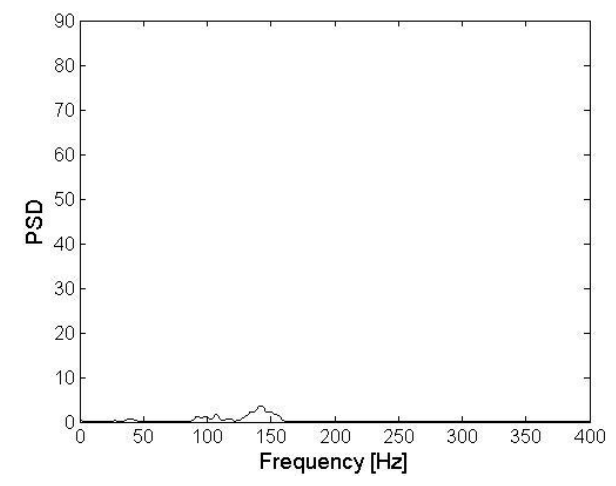

b. PSD on VKF-OT for $3^{\text {rd }}$ order

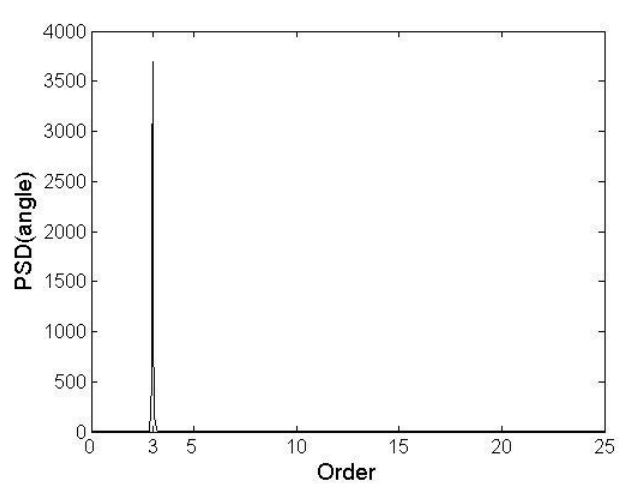

d. PSD on VKC-OT for $3^{\text {rd }}$ order

Figure 4 PSD results for scenario 2 (with noise)

Little can be learnt from the spectrum in Figure 4(a) because of the noise. Figure 4(b) and 4(c) show the PSD and PSD(angle) after VKF-OT and COT respectively, and do give some insight into the system, albeit limited compared to Figure 3. It should also be noted that the energy in Figure 4(b) is far lower than that in Figure 4(a). This indicates that the VKF-OT technique largely excludes the influence of noise and focuses on the order of interest. Finally, however, Figure 4(d) provides another clear $3^{\text {rd }}$ order peak, largely excluding the influence of noise and non-stationarity. This result further demonstrates that the combined use of VKF-OT and COT can significantly enhance the result obtained from Fourier analysis.

\section{Experimental results}

\subsection{Rotating electrical machinery}

Wang and Heyns [11] established an experimental test rig to investigate the ability of VKF-OT in condition monitoring. Typical run-up data (vibration and tachometer signals) from an automotive alternator were obtained. The experimental setup is shown in Figure 5. 


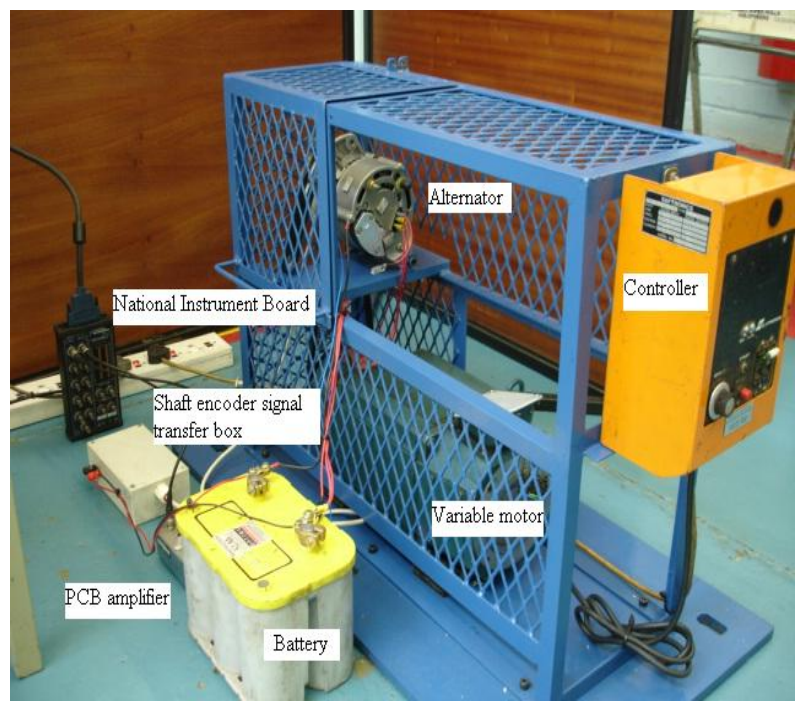

Figure 5 Automotive alternator set-up

A typical set of measured data and the corresponding rotational speed are plotted in Figure 6, and show how the vibration as well as the speed changes with time.
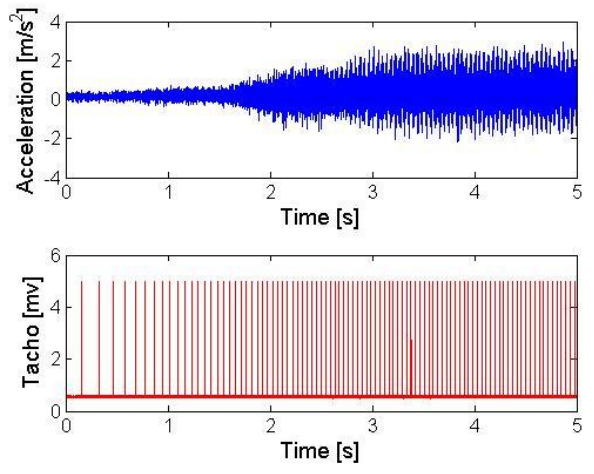

a. Measured data

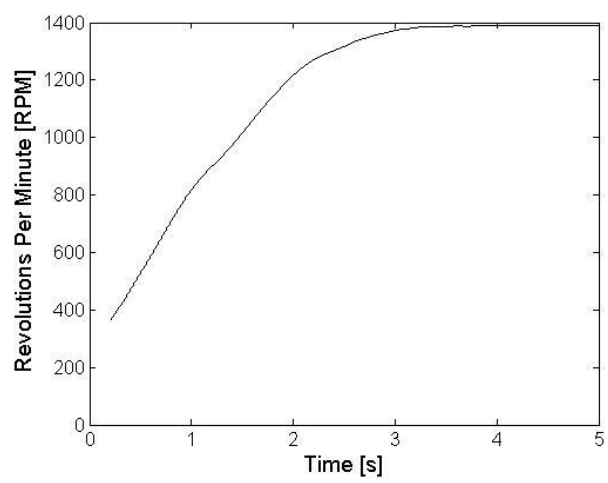

b. RPM

Figure 6 Raw data set

Using this data, the same techniques as were discussed in the SDOF rotor simulation study are again applied here, and the four PSDs are plotted in Figure 7. Since there are 36 stator bars in the alternator, the Vold-Kalman filter is used here to extract the $36^{\text {th }}$ order. 


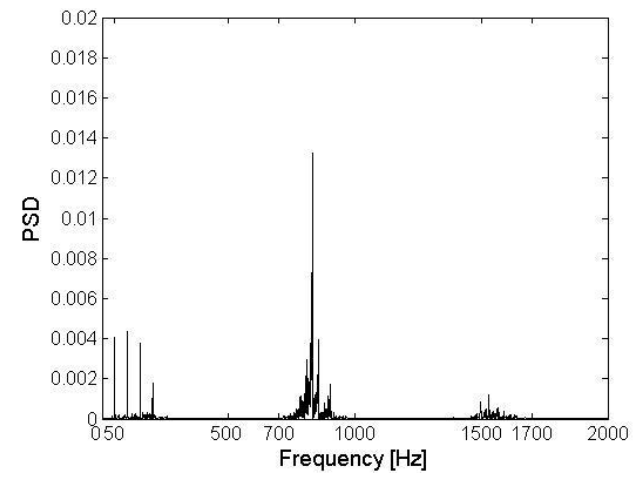

a. PSD on raw data

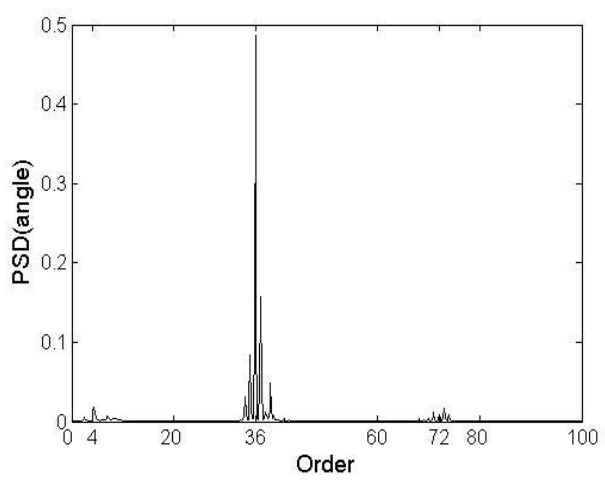

c. PSD on COT data

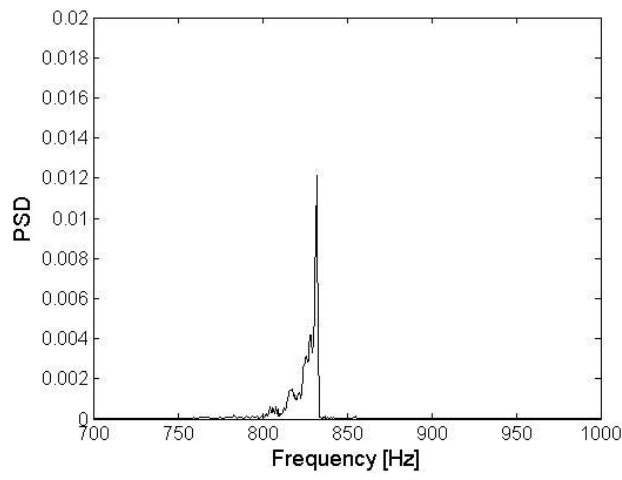

b. PSD on VKF-OT for $36^{\text {th }}$ order

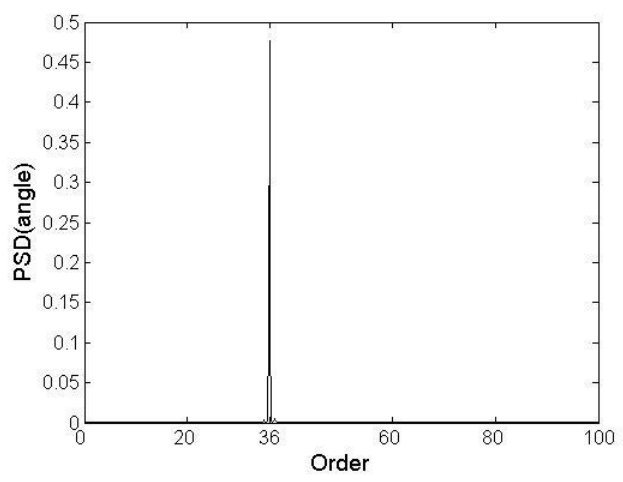

d. PSD on VKC-OT for $36^{\text {th }}$ order

Figure 7 PSD results for alternator data

Figure 7(a) shows the PSD of the raw data. There are several frequency peaks at around 50, 100 and $150 \mathrm{~Hz}$, and some higher-frequency peaks around $700-1000 \mathrm{~Hz}$, and $1500-1700 \mathrm{~Hz}$. Since the alternator output frequency is $50 \mathrm{~Hz}$, it is expected to have frequencies at $50 \mathrm{~Hz}$ and its multiples. Besides, based upon the rotating speed, the full speed of the alternator nearly approached $1400 \mathrm{RPM}$ or $\approx 23.3 \mathrm{~Hz}$. Considering the physical structure of the alternator, namely that there are 36 stator bars, therefore $36 \times 23.3=838.8 \mathrm{~Hz}$ or its multiples may appear, i.e. $72 \times 23.3=1677.6 \mathrm{~Hz}$. These two frequencies are apparently within these higher-frequency peaks. It is difficult however to obtain any definite information from Figure 7(a). Then, if Vold-Kalman filter-based order tracking is performed with a relative filter bandwidth of $30 \%$, the associated PSD spectrum is plotted in Figure 7 (b) for the $36^{\text {th }}$ order. In order to see the filtered $36^{\text {th }}$ order clearly, the abscissa zoomed in the range from $700 \mathrm{~Hz}$ to $1000 \mathrm{~Hz}$. Order 36 is now much clearer than in Figure 7(a), but the smearing effect is still present and it is still not ideal for monitoring purposes. Figure 7(c) is the PSD(angle) of the COT result. It can be clearly seen that order 36 and its sideband orders are all included in the figure and also the lower orders at around 4 as well as higher orders at around 72. It is quite clear however that it cannot focus on one order, so it features several order peaks in the figure, which is not ideal for the purpose of condition monitoring either. Finally, 
VKC-OT is applied to the data to extract the $36^{\text {th }}$ order in Figure 7(d). It can be seen that a clear and clean $36^{\text {th }}$ order peak exists, which effectively excludes other sideband orders as well as the smearing effect.

\section{Conclusion}

Vold-Kalman filter-based order tracking and computed order tracking have different advantages and limitations. The distinct advantage of the re-sampling process of COT is its ability to transform non-stationary data into stationary data, but it does nothing with the data to eliminate the imperfections of the data and does not have the ability to focus on individual orders. One of the main limitations of VKF-OT is that the filtered data will retain the non-stationary effect of the raw data, but the filtering process enforces smoothness and the harmonic nature of the data, which are important factors for meaningful Fourier analysis. Through the combined use of Vold-Kalman filter and computed order tracking (VKC-OT), the limitations of Fourier analysis are largely avoided. The benefits of VKC-OT are demonstrated by a simulated SDOF rotor model and the measured data from an automotive alternator. Both studies confirmed the advantages of VKC-OT in order tracking analysis.

In short, a novel approach VKC-OT has been presented in this paper. The technique takes the advantages of VKF-OT in its ability to extract focused, smooth and harmonic nature order waveform and of COT in its ability to transform non-stationary time domain data into stationary angle domain data, so that the novel VKC-OT method provides a clear and focused perspective for the analyst to examine order components by using simple Fourier analysis which would be an intractable problem when using any other order tracking techniques alone.

\section{References}

[1] Fyfe, K. R. and Munck, E. D. S., Analysis of computed order tracking. Mechanical Systems and Signal Processing, 11(2), 1997, pp.187-205.

[2] Eggers, B. L., Heyns, P. S. and Stander, C. J., Using computed order tracking to detect gear condition aboard a dragline. The Journal of Southern African Institute of Mining and Metallurgy, 107, 2007, pp.1-8.

[3] Brüel and Kjær, Product Data: Vold-Kalman order tracking filter - Type 7703 for Pulse. Available at: http://www.bksv.com/pdf/bp1760.pdf/ [Accessed: 11 Sep 2007.]

[4] Vold, H., Mains, M. and Blough, J., Theoretical foundations for high performance order tracking with the Vold-Kalman tracking filter. SAE Paper 972007, 1997, pp.1083-1088.

[5] Tůma, J., Setting the pass bandwidth in the Vold-Kalman order tracking filter. Twelfth International Congress on Sound and Vibration, 11-14 July 2005, Lisbon.

[6] Pan, M. Ch. and Lin Y. F., Further exploration of Vold-Kalman filtering order 
tracking with shaft-speed information-I: Theoretical part, numerical implementation and parameter investigations. Mechanical Systems and Signal Processing 20(5), 2006, pp.1134-1154.

[7] Herlufsen, H., Gade, S. and Konstantin-Hansen, H., Characteristics of the Vold/Kalman order tracking filter. Proceedings of the $17^{\text {th }}$ International Modal Analysis Conference, Kissimmee, FL, March 1999.

[8] Vibrotools in MATLAB. Available at :http://www.vibratools.com/ [Accessed: 11 Sep 2007.]

[9] Howell, K. B., Principles of Fourier analysis, Chapman \& Hall/CRC, 2001.

[10] Blough, J. R., A survey of DSP methods for rotating machinery analysis, what is needed, what is available. Journal of Sound and Vibration, 262, 2003, pp.707-720.

[11] Wang, K. S. and Heyns, P. S., Vold-Kalman filter order tracking in vibration monitoring of electrical machines. Journal of Vibration and Control, 2009, 15(9), pp.1325-1347. 